Article

\title{
Variant Selection in Fe-20Ni-1.8C under Bending
}

\author{
Annick P. Baur *, Cyril Cayron and Roland E. Logé
}

Laboratory of Thermomechanical Metallurgy-PX Group Chair, Institute of materials, Ecole Polytechnique Fédérale de Lausanne, 2000 Neuchâtel, Switzerland; cyril.cayron@epfl.ch (C.C.); roland.loge@epfl.ch (R.E.L.)

* Correspondence: annick.baur@epfl.ch; Tel.: +41-2-1695-4481

Received: 15 November 2018; Accepted: 13 December 2018; Published: 18 December 2018

\begin{abstract}
Variant selection is commonly observed in martensitic steels when a stress is applied to the material during transformation. Classically, the selection phenomenon is modelled considering the work of the shape strain in the applied stress field. This shape strain is generally calculated by using the Phenomenological Theory of the Martensite Crystallography (PTMC). In the present study, we studied the martensitic transformation occurring in a Fe-20wt $\% \mathrm{Ni}-1.8 \mathrm{wt} \% \mathrm{C}$ alloy transformed while loaded in four-point bending. A significant variant selection is observed, but surprisingly its nature cannot be explained by the classical approach. A crystallography-based empirical model which accounts for the experimental results is proposed instead.
\end{abstract}

Keywords: martensite; variant selection; PTMC

\section{Introduction}

The effect of applied stress on the martensitic transformation has been studied for almost one century, Scheil being one of the first researchers who worked on this topic in 1932 [1]. In 1953, Patel and Cohen [2] performed a series of experiments on iron-nickel alloys and proposed a physical criterion to quantify the effect. The criterion allows to calculate the change of the martensitic start temperature as a function of the stress applied to the material. In their study, Patel and Cohen showed that if both the application of a uniaxial compressive stress and a uniaxial tensile stress increase the temperature at which martensite forms, the increase due to the latter is more significant. Accordingly, they proposed a criterion for martensite formation based on the work of the shape strain associated with the transformation in the applied stress field. This criterion is now known as the Patel-Cohen criterion. The shape strain considered is an invariant plane strain, where the invariant plane is the interface between martensite and austenite (habit plane). It can be calculated for any alloy by using the Phenomenological Theory of Martensite Crystallography (PTMC). The PTMC is probably the most famous crystallographic model for the martensitic phase transformation. It is the result of the work of two independent groups of researchers: Liebermann, Wechsler and Read in 1953 [3], and Bowles and Mackenzie in 1954 [4]. Both chose to model the transformation using linear algebra, but they came up with two apparently different expressions. A couple of years later, Christian showed that the two models were in fact equivalent [5]. Expressed in the Bowles and Mackenzie version, the transformation is modelled using four different matrices, each of them accounting for a part of the transformation. The structural phase change and the orientation relationship are characterized respectively, by the Bain strain $\mathbf{B}$ [6] and a rotation $\mathbf{R}$, and their product $\mathbf{R} \mathbf{B}$ gives the lattice strain. The shape change is described by the matrix $\mathbf{P}$, which is the shape strain. The last matrix $\mathbf{Q}$ renders compatible the shape strain with the lattice strain. It is named the lattice invariant shear. The PTMC stands then in a single equation $\mathbf{R B}=\mathbf{P Q}$. After its publication, this model quickly became very popular and remains as such today. The PTMC calculations allow in particular to study variant selection using the predicted shape strain to evaluate the Patel-Cohen criterion. Such an approach is broadly accepted and commonly 
used $[7,8]$. However, some experiments indicate that this criterion is not appropriate to explain the phenomenon. As an example, Mishiro et al. applied a compressive stress just above the martensitic start temperature to induce variant selection in a low-carbon steel. They noted that the lattice strain RB better accounts for the selection than the shape strain $\mathbf{P}$ [9]. Chiba et al. reported a significant variant selection in Fe-Ni-C lenticular martensite formed after ausforming [10]. They observed that four variants were preferentially formed in each parent grain. They applied the Patel-Cohen criterion using the residual stress to account for variant selection, but this analysis was not satisfactory. Indeed, the experimentally observed variants had their habit plane oriented rather perpendicularly to the compression direction and the resolved shear stress acting on them was very low. They concluded that variant selection was not related to the residual stress, but to the elongated morphology of the prior austenite grains. Such morphology favours the variants having a habit plane oriented parallel to the elongated direction as they induce less disturbance at the austenite gain boundary.

In the present study, we observe similar results in lenticular martensite formed in $\mathrm{Fe}-20 \mathrm{wt} \% \mathrm{Ni}-1.8 \mathrm{wt} \% \mathrm{C}$ under bending. A strong variant selection is measured, and the selection cannot be explained convincingly by the PTMC. The phenomenon is eventually accounted by an empirical crystallographic criterion.

\section{Material and Methods}

A Fe-20wt $\% \mathrm{Ni}-1.8 \mathrm{wt} \% \mathrm{C}$ alloy has been homogenized at $1000{ }^{\circ} \mathrm{C}$ for $1 \mathrm{~h}$ in a quartz tube. After the heat treatment, the material is directly quenched in water. A thin rectangular sample is cut out of the material. The sample is loaded in a miniaturized home-designed four-point bending device. The device is illustrated in Figure 1A. The loading is performed by moving the two central pins down, using a vertical screw. Such a loading system imposes a compression in the upper part of the sample and a tension in the lower part. The line in the middle of the sample thickness is called neutral line, as no stress acts on it. Martensitic phase transformation is induced in the sample by cooling it to $-80^{\circ} \mathrm{C}$ in a freezer. The specimen is maintained in the device during transformation. The imposed stress thus interacts with the transformation mechanism. The four-point bending device is designed such that the deformed sample can be directly embedded for material characterization, while keeping its exact shape. Figure 1B shows the embedded sample. The sample is prepared for EBSD characterization by mechanical grinding followed by a final polishing using Vibromet table. In order to study variant selection, some EBSD measurements have been performed in a XLF-30 (FEI) scanning electron microscope, equipped with a Channel5/Aztec (Oxford Instruments) EBSD system.

A

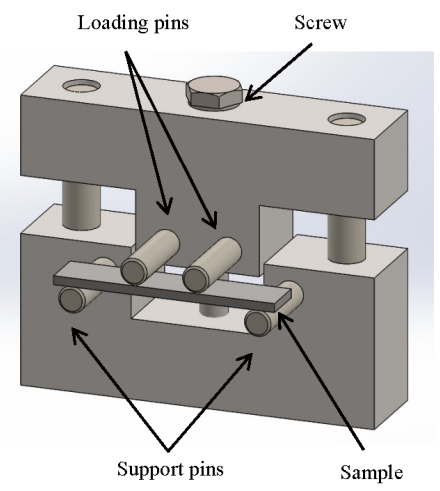

B

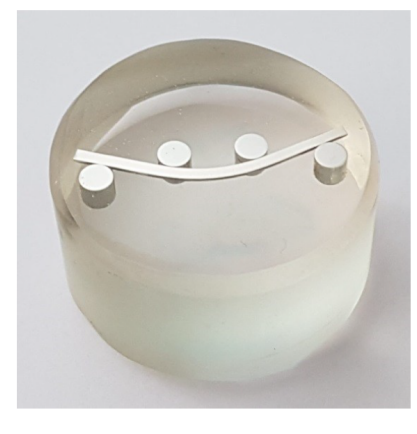

Figure 1. (A) Four-point bending device. (B) Bended sample embedded in epoxy for material characterization.

\section{Results}

Figure 2 shows an optical micrograph of the microstructure after transformation. The two pins in the upper corners of the picture are the central loading pins of the 4-point bending setup. It can be seen that the transformation only takes place in the tensile part of the sample. The propensity for the martensite to form preferentially in the tensile part of a bended sample was already observed by Kulin, 
Cohen and Averbach in three-point bending [11]. A few years later, this observation was rationalized by the aforementioned Patel-Cohen criterion [2]. It appears that our alloy is such that at $-80^{\circ} \mathrm{C}$ only the part in tension has enough driving force to induce the martensitic transformation. According to the $\mathrm{Fe}-\mathrm{Ni}-\mathrm{C}$ phase diagram, the heat treatment at $1000{ }^{\circ} \mathrm{C}$ leads to the dissolution of only $0.85 \mathrm{wt} \%$ of the carbon, the rest is forming the carbides visible in Figure 2. The Ms reported for a Fe-20wt $\% \mathrm{Ni}-0.8 \mathrm{wt} \% \mathrm{C}$ is $-74^{\circ} \mathrm{C}$ [12]. Considering our slightly higher carbon content, a transformation temperature close to $-80{ }^{\circ} \mathrm{C}$ is reasonable. As an interesting feature, it can be noted that the transformation is localized and takes place in "columns", the columns stopping near to the neutral line. Figure 3 shows the EBSD measurement of a column of martensite. This measurement indicates that only a few variants are formed in each austenitic parent grain, suggesting a probable variant selection phenomenon.

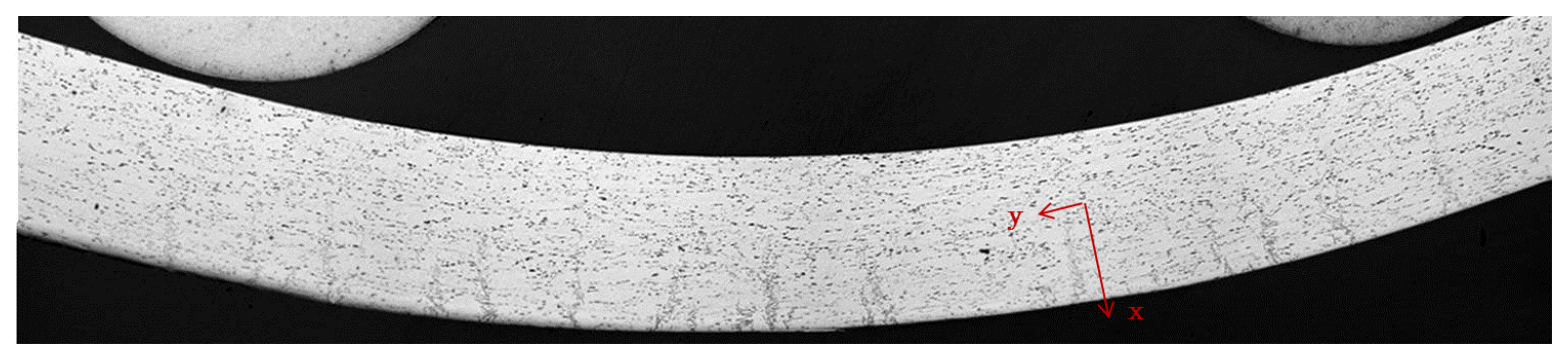

Figure 2. Bright-field optical micrograph of the sample in 4-point bending. The axis in red indicates the orientation of the EBSD measurement.

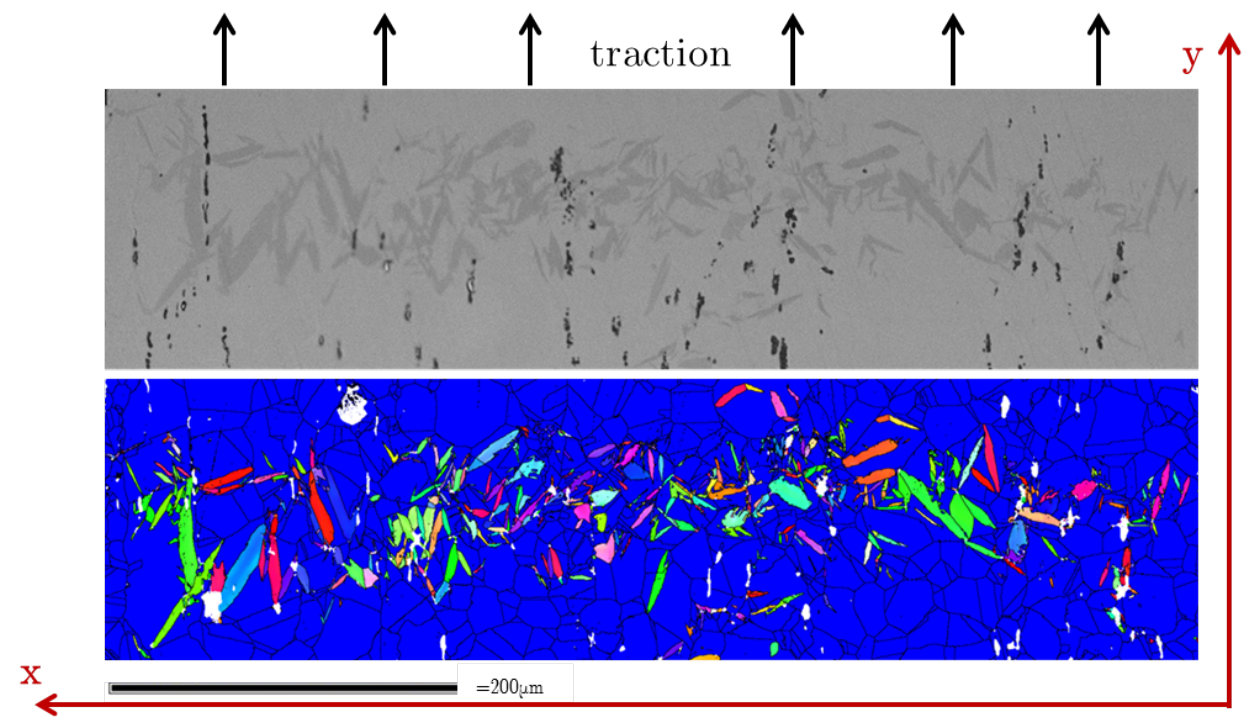

Figure 3. EBSD map of a column of martensite with the austenite in blue and the martensite in Euler colour coding and below, bright-field optical micrograph of the corresponding zone. The black arrows indicate the traction direction and the red axis can be related to those in Figure 2.

\section{Discussion}

To address variant selection, two different PTMC calculations are performed using the PTCLab open-source software [13]. The first calculation considers an FCC-BCC transformation and the second an FCC-BCT transformation, including the tetragonality $\gamma=\frac{c_{\alpha}}{a_{\alpha}}=1.045$ observed in Fe-Ni-C alloys of close compositions [12,14]. The lattice parameters $a_{\alpha}=2.87 \AA$ and $a_{\gamma}=3.58 \AA$, and $a_{\alpha}=2.87 \AA$, $c_{\alpha}=3.00 \AA$ and $a_{\gamma}=3.58 \AA$ measured in similar alloys are the inputs for the calculations of the BCC and the BCT models, respectively [15]. The simple shear version of the PTMC is used and the lattice invariant shear is chosen as suggested in the Bowles and Mackenzie original theory for the Fe-Ni-C martensite, namely a "twinning shear" of type $<111>\{112\}_{\alpha}$ [4]. The shape strain $\mathbf{P}$ 
and $\mathbf{P}_{\mathrm{T}}$ and lattice strain $\mathbf{R B}$ and $\mathbf{R} \mathbf{B}_{\mathrm{T}}$ matrices are given below for the FCC-BCC and the FCC-BCT transformations, respectively:

$$
\begin{array}{lll}
\mathbf{P} & =\left[\begin{array}{ccc}
0.99264 & 0.03316 & -0.02733 \\
-0.02641 & 1.119 & -0.09808 \\
-0.02733 & 0.12315 & 0.8985
\end{array}\right] & \mathbf{R B}=\left[\begin{array}{ccc}
1.11731 & 0.03316 & 0.09733 \\
-0.01765 & 1.119 & -0.08932 \\
-0.14049 & 0.12315 & 0.78534
\end{array}\right] \\
\mathbf{P}_{\mathbf{T}}=\left[\begin{array}{ccc}
0.96057 & -0.07824 & -0.02312 \\
0.06557 & 1.13011 & 0.03844 \\
-0.02312 & -0.04587 & 0.98645
\end{array}\right] & \mathbf{R B}_{\mathbf{T}}=\left[\begin{array}{ccc}
1.11664 & -0.07824 & 0.13296 \\
0.06987 & 1.13011 & 0.04274 \\
-0.18329 & -0.04587 & 0.82627
\end{array}\right]
\end{array}
$$

In both cases, the orientation relationship predicted by the PTMC is between the Nishiyama-Wassermann OR and the Kurdjumov-Sachs OR. The calculated habit planes are $\{0.170 .630 .76\}_{\gamma}$ for the BCC model and $\{0.260 .440 .86\}_{\gamma}$ for the BCT. These habit planes are respectively at $10^{\circ}$ and $5^{\circ}$ from $\{259\}_{\gamma}$ and $6^{\circ}$ and $9^{\circ}$ from $\left\{\begin{array}{lll}3 & 10 & 15\end{array}\right\}_{\gamma}$, which are the typical habit planes observed in such alloys [16]. Variant selection is studied using the Patel-Cohen criterion for martensite formation, as suggested in the literature [7]. The variants selected are the ones that maximize the work $W$ of the shape strain $\mathbf{P}$, respectively $\mathbf{P}_{\mathbf{T}}$, in the applied stress field $\sigma ; W=\epsilon_{i j} \sigma_{i j}$ with $\epsilon=\mathbf{P}-\mathbf{I}$ and $\sigma$ is the generalized stress tensor associated with the external loading. In four-point bending, the generalized stress tensor is reduced to a single non-zero entry, as the material is either in simple compression or simple traction. Here, we choose to orientate the specimen such that the traction/compression axis is aligned with the y-axis. Accordingly, the stress tensor is $\sigma_{i j}=0$ if $i, j \neq 2$. For each austenitic grain, the daughter variants are classified in respect with the value of their work. The variant exhibiting the maximal work is ranked first (1) and the one with the lowest work is ranked last (24). The calculations are performed using the Mtex toolbox [17]. Figure 4A,B present the histograms of the variant occurrence in the EBSD map according to the proposed ranking for the BCC and the BCT models. The occurrence of a given variant is measured by counting the number of pixels associated with the variant.

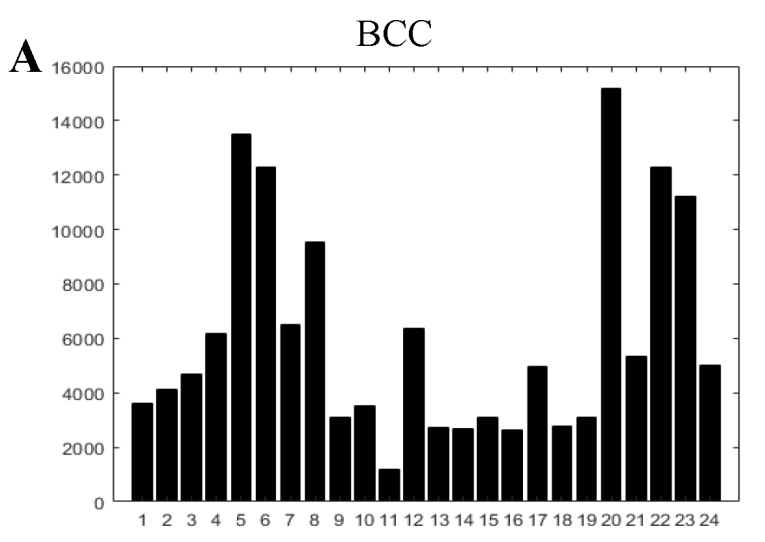

High work

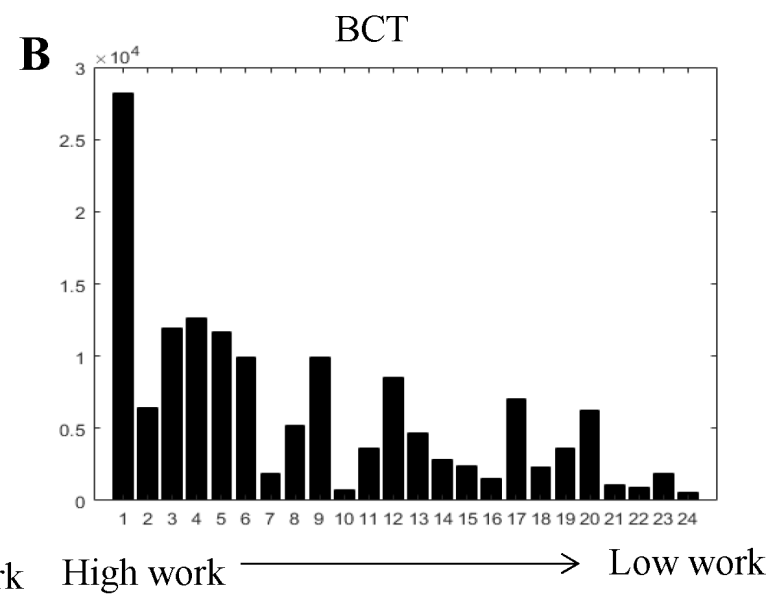

Figure 4. Histograms of the variants' occurrence considering the BCC model in (A) and the BCT model in (B). In the $y$-axis: the number of pixels associated with a given variant number. In $x$-axis: variants numbered with a ranking according to the value of the work.

With such a ranking, if the variant selection is based on the work of the shape strain, one expects that the variant with a small number, and thus exhibiting high work, would be observed more often than those associated with greater numbers. On this basis, it can be concluded that the present experiment does not exhibit a variant selection based on the work of the BCC shape strain, as there is no correlation between the ranking of the variants and their occurrence. On the contrary, the BCT 
model seems to correctly predict the variant selection, at least for the first variant, i.e., the one with the highest work. A verification of the habit plane prediction is then performed by studying an austenite grain selected from the EBSD map presented in Figure 3. The grain is shown in Figure 5A with the austenite in blue and the martensite in Euler color coding. In Figure $5 B, C$ respectively, the shape strains predicted by the PTMC calculations for the BCC and BCT models are shown for all four variants in Figure 5A. The normals to the predicted habit planes are marked in green and the shear directions are marked in yellow. Numbers are given to each variant to help in associating them with their shape strain.

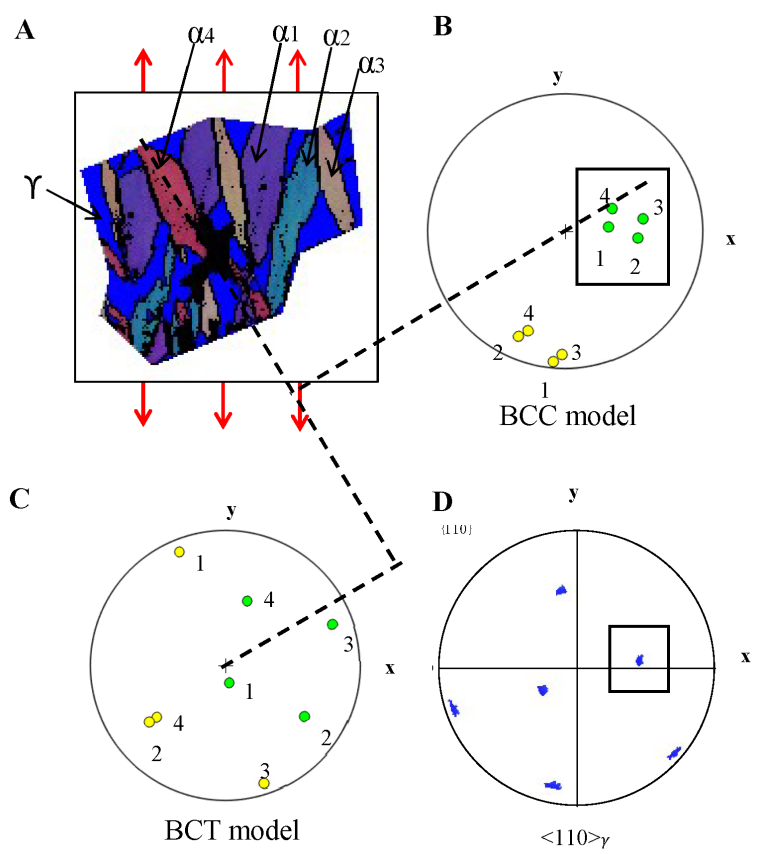

Figure 5. Trace analysis of the shape strain predicted by the Phenomenological Theory of Martensite Crystallography (PTMC) and variant selection prediction. (A) EBSD map of the grain with red arrows indicating the traction direction. The dashed lines indicate the normal to the habit plane of variant $\alpha_{4}$. (B) Pole figure of the normals to the habit planes (green) and the shear directions (yellow) predicted by the BCC model. (C) Pole figure of the normal to the habit planes (green) and the shear directions (yellow) predicted by the BCT model. (D) $<110>_{\gamma}$ experimental pole figure.

The midrib is considered as the habit plane of the martensite and a trace analysis is performed to verify the PTMC predictions. As an example, the normal to the habit plane of the red variant $\alpha_{4}$ is marked with dashed lines in Figure 5. Our analysis clearly indicates that the BCT model does not predict the observed habit plane. This model which seemed to correctly account for variant selection is thus not satisfactory. On the contrary, the BCC model which fails to account for the variant selection seems to correctly predict the habit planes. From Figure 5, we can see that selected habit planes almost contain the traction direction. Hence, the shear resolved on it is almost zero, which explains why the criterion based on the work of the shape strain associated with the BCC model is not able to account for the variant selection. To be exhaustive in our variant selection analysis, we also computed the work of the elastic part of the BCC shape strain using $\epsilon=\frac{1}{2}\left(\mathbf{P}+\mathbf{P}^{T}\right)-\mathbf{I}$, the work of the lattice strain $\mathbf{R B}$ and the work of the lattice invariant shear $\mathbf{Q}$. Additionally, we considered the couplings of variants inside the same plate group by computing the work of the average strain resulting from different shape strains, as suggested by Okamoto et al. [18]. None of these criteria accounts for the variant selection observed in the sample. These features are somewhat coherent with the results presented by Chiba et al. on variant selection in lenticular martensite [10]. They also observed a lack of correlation between such energetic criteria and the measured variant selection. In agreement with our 
observations, they noted that the favoured variants have their habit planes normal to the compression axis, with low resolved shear acting on them. They concluded that the selection phenomenon was related to the elongated morphology of the prior austenite grain. In our sample, the parent austenite grains have a quite equiaxed geometry and Chiba et al.'s explanation is thus not applicable. It seems, however, that even though the thermomechanical treatments are different, the results of Chiba et al. have similarities with ours. As illustrated in Figure 5A, we also observe that four variants form preferentially, these variants belonging to the same plate group. The variant coupling in plate groups is frequently observed in lenticular martensite [19-21]. The plate groups were originally observed by Bokros and Parker in Fe-31\%Ni [21]. They are defined in terms of habit plane orientation, as the group formed by the variants having their habit planes that cluster around a given $\langle 110\rangle_{\gamma}$ direction of the parent crystal. An illustration of such a cluster is proposed in Figure 5D,B where squares are drawn to indicate the $<110>_{\gamma}$ direction and the associated habit plane normals, respectively. In terms of lattice orientation, the plate group is crystallographically defined in reference [20] and illustrated with the yellow Kurdjumov-Sachs variants in Figure 6A. This illustration is adapted from Figure 4E of ref. [20]. The parent austenite is represented by two of the $\{111\}_{\gamma}$ planes, and the two others are not visible. The Kurdjumov-Sachs variants are illustrated by the green and yellow rectangles, representing the $\{110\}_{\alpha} \|\{111\}_{\gamma}$. The yellow rectangles represent one of the six possible plate groups. According to this definition, the plate group can be defined as the variants that have their $\langle 111\rangle_{\alpha}$ that scatter about $10^{\circ}$ from a $<110>_{\gamma}$. It should be noted that the $<110>_{\gamma}$ direction that defines the plate group in terms of the habit plane or in terms of lattice orientation are different. In Figure 6A,B, we marked in purple the considered $<111>_{\alpha}$ and in red the associated $<110>_{\gamma}$.

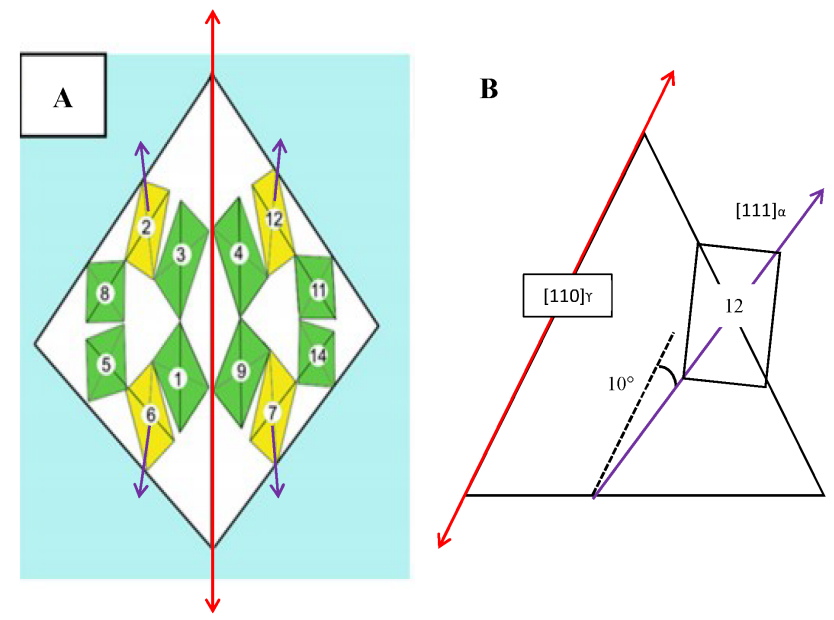

Figure 6. Empirical criterion for variant selection. (A) Plate group represented in 3D on two $\{111\}_{\gamma}$ planes. Illustration adapted from ref. [20]. (B) Triangle representing the $(1 \overline{1} 1)_{\gamma}$ plane and rectangle representing in $2 \mathrm{D}$ the $(1 \overline{1} 0)_{\alpha}$ of variant 12 . In purple, the $[111]_{\alpha}$ that clusters about the $[110]_{\gamma}$ marked in red.

In our experiment, a single plate group is formed preferentially in each parent grain. Based on this observation, we were able to express an empirical criterion to characterize the plate group that is favoured under traction. This criterion cannot distinguish the different variants inside the same group and thus does not treat the more severe selection that can be observed in some grains, where only one or two variants out of the four appear. Our criterion states that the selected plate group is the one formed by the four variantshaving their $<111>_{\alpha}$ that cluster about the $<110>_{\gamma}$ direction which is closest to the traction axis. In Figure 6A, the yellow plate group would be selected if the traction axis is close to the red arrows. Based on that empirical rule, we classify the plate groups as a function of the angle between the traction direction and the $\langle 110\rangle_{\gamma}$ direction associated with each plate group. The plate group with the lowest angle is ranked first (1) and the plate group with the largest angle is 
ranked last (6). Figure 7 shows the histogram of variant occurrence according to the proposed criterion for the entire column of martensite shown in Figure 3.

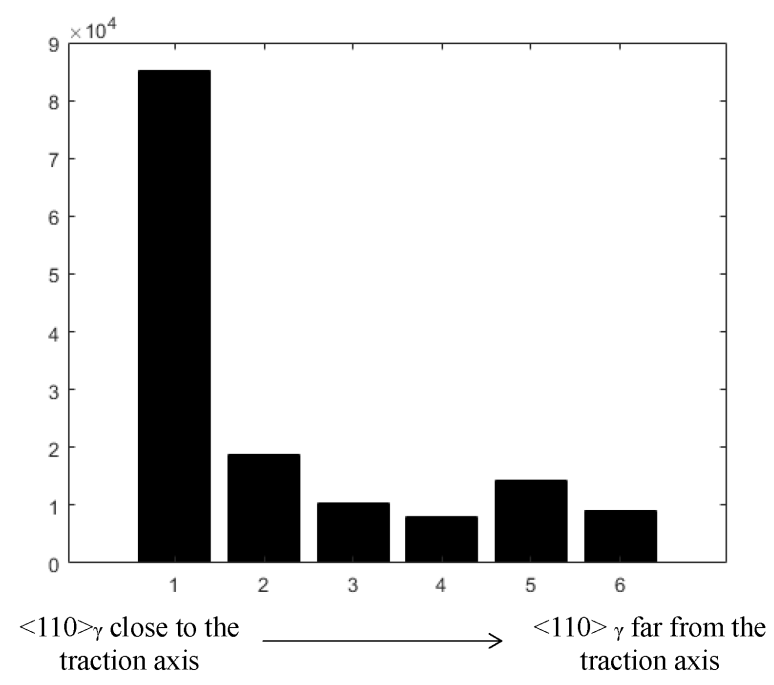

Figure 7. Histograms of plate group occurrence considering a ranking based on the empirical criterion. In the $y$-axis: the number of pixels associated with a given plate. In the $x$-axis: plate group numbered with a ranking according to the empirical criterion.

It appears that this criterion accounts very well for the variant selection observed in our sample. Figure 8 illustrates the selection phenomenon for the grain in Figure 5A with the austenite in blue and the martensite in Euler color coding. Figure 8A presents the experimental pole figures of the grain. Figure 8B shows all Kurdjumov-Sachs variants with empty markers and the variants selected by our criterion with blue markers. Figure $8 \mathrm{C}$ shows the orientation of the parent grain. We marked with a circle the $<110>_{\gamma}$ direction about which the $<111>_{\alpha}$ of the variants of the selected plate group clusters. The circle is reported on the martensite pole figures, in Figure 8A,B, to indicate the $<111>_{\alpha}$ cluster. 


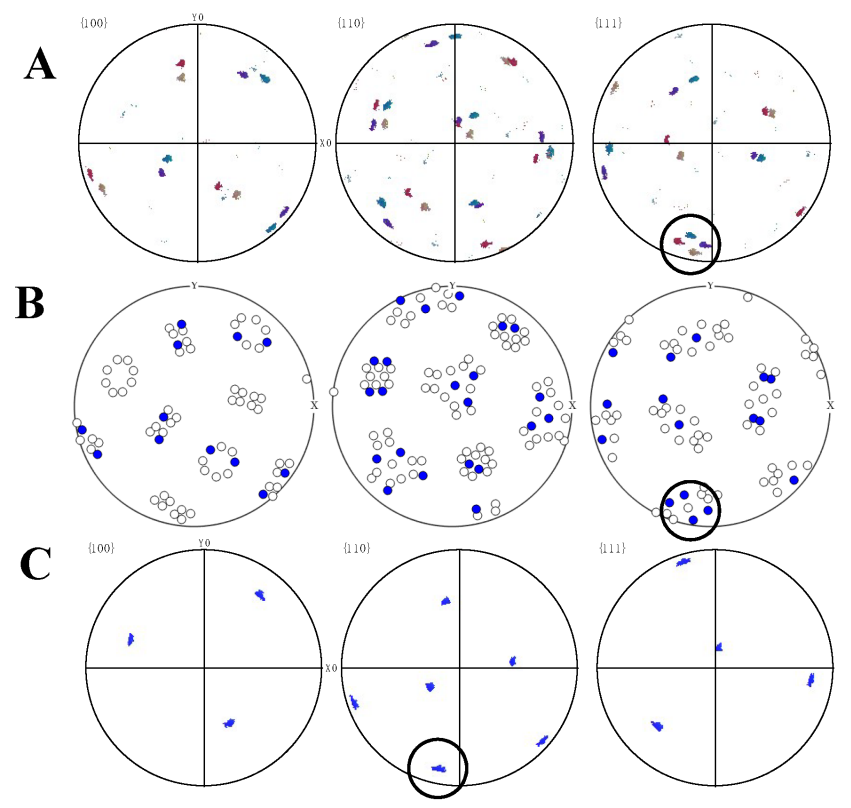

Figure 8. Variant selection in the single parent grain presented in Figure 5A. The circle indicates that the $<111>_{\alpha}$ scatters about the $<110>_{\gamma}$ that is closest to the traction axis which is oriented along the y-direction. (A) Experimental pole figures of the martensite. (B) Kurdjumov-Sachs pole figures with the variant selected by our criterion marked in blue. (C) Experimental pole figures of the parent austenite.

It is quite puzzling to see that even though the BCC model prediction of the orientation relationship and the habit plane orientation are in fairly good agreement with the experimental measurements, the variant selection based on the work of the shape strain does not hold, and that on the contrary, the BCT model accounts for the variant selection but considering a shape strain which is not observed. It seems that the mechanism of the formation of lenticular martensite is not fully understood and that its current modeling is not able to depict all the physics involved in the transformation. The nature of the lattice invariant shear in particular seems to be controversial. The PTMC theory considers that a single shear occurs to accommodate the $\{259\}_{\gamma}$ martensite. However, Thomas observed that more than one lattice-invariant shear effectively acts in the accommodation process [22].

In conclusion, variant selection has been studied in a Fe-20Ni-1.8C alloy transformed under bending. The transformation takes place only in the tensile part of the specimen and a significant variant selection is observed. It appears that a single plate group is formed preferentially in each grain. The variant selection is studied using the Patel-Cohen criterion with two different shape strains associated with the FCC-BCC and the FCC-BCT transformations. The shape strains are computed using PTMC. The BCT model is shown to correctly account for the variant selection, but the shape strain does not agree with the experimental data. In contrast, the BCC model predicts well the habit plane orientation, but is not able to explain the variant selection. These discrepancies between the experiment and PTMC predictions need to be further analyzed in order to better understand the mechanism of formation of the lenticular martensite and possibly explain what drives the observed variant selection. An empirical criterion, which captures the selection, is proposed. However, the physical explanation for such a criterion remains mysterious and more advanced characterization techniques to analyze the martensite substructure might be required to rationalize it.

Author Contributions: Conceptualization, A.P.B., C.C. and R.E.L.; Methodology, A.P.B. and C.C.; Software, A.P.B.; Validation, A.P.B. and C.C.; Formal analysis, A.P.B. and C.C.; Investigation, A.P.B.; Resources, A.P.B. and C.C.; Data curation, A.P.B.; Writing-original draft preparation, A.P.B., C.C. and R.E.L.; Writing-review and editing, A.P.B., C.C. and R.E.L.; Visualization, A.P.B.; Supervision, C.C. and R.E.L.; Project administration, C.C.; Funding acquisition, C.C. and R.E.L.

Funding: This research was funded by the Swiss National Science Foundation (grant number 200021-159955). 
Acknowledgments: The authors would like to thank Mathijs van der Meer for its help in designing the four-points bending device. The authors also acknowledge ArcelorMittal, in particular Ian Zuazo and Kangying Zhu for providing the material of this study. Finally, the authors would like to thank PX Group for its financial support to the laboratory.

Conflicts of Interest: The authors declare no conflict of interest.

\section{References}

1. Scheil, E. Uber die Umwandlung des Austenits in Martensit in Eisen-Nickellegierungen unter Belastung. Z. Anorg. Allg. Chem. 1932, 207, 21-40. [CrossRef]

2. Patel, J.; Cohen, M. Criterion for the action of applied stress in the martensitic transformation. Acta Metall. 1953, 1, 531-538. [CrossRef]

3. Wechsler, M.S. Lieberman, D.; Read, T. On the theory of the formation of martensite. Trans. AIME 1953, 197, 1503-1515.

4. Bowles, J.; Mackenzie, J. The crystallography of martensite transformations I. Acta Metall. 1954, 2, $129-137$. [CrossRef]

5. Christian, J. Applications of the phenomenological theories of martensite I-II. J. Inst. Met. 1956, 84, $386-394$.

6. Bain, E.C.; Dunkirk, N. The nature of martensite. Trans. AIME 1924, 70, 25-47.

7. Kundu, S.; Bhadeshia, H. Crystallographic texture and intervening transformations. Scr. Mater. 2007, 57, 869-872. [CrossRef]

8. Marechal, D.; Malet, L.; Godet, S.; Sinclair, C.W. Variant Selection and Texture in an AISI 301LN Stainless Steel. In Materials Science Forum; Trans Tech Publications: Princeton, NJ, USA, 2012; Volume 702, pp. 850-853.

9. Mishiro, Y.; Nambu, S.; Inoue, J.; Koseki, T. Effect of stress on variant selection in lath martensite in low-carbon steel. ISIJ Int. 2013, 53, 1453-1461. [CrossRef]

10. Chiba, T.; Miyamoto, G.; Furuhara, T. Variant selection of lenticular martensite by ausforming. Scr. Mater. 2012, 67, 324-327. [CrossRef]

11. Kulin, S.; Cohen, M.; Averbach, B. Effect of applied stress on the martensitic transformation. JOM 1952, 4, 661-668. [CrossRef]

12. Kajiwara, S.; Kikuchi, T. On the abnormally large tetragonality of martensite in Fe-Ni-C alloys. Acta Metall. Mater. 1991, 39, 1123-1131. [CrossRef]

13. Gu, X.F.; Furuhara, T.; Zhang, W.Z. PTCLab: free and open-source software for calculating phase transformation crystallography. J. Appl. Crystallogr. 2016, 49, 1099-1106. [CrossRef]

14. Greninger, A.B.; Troiano, A.R. The mechanism of martensite formation. JOM 1949, 1, 590-598. [CrossRef]

15. Reed, R.; Schramm, R. Lattice parameters of martensite and austenite in Fe-Ni alloys. J. Appl. Phys. 1969, 40, 3453-3458. [CrossRef]

16. Nishiyama, Z. Martensitic Transformation; Elsevier: Amsterdam, The Netherlands, 2012.

17. Bachmann, F.; Hielscher, R.; Schaeben, H. Texture analysis with MTEX-free and open source software toolbox. In Solid State Phenomena; Trans Tech Publications: Princeton, NJ, USA, 2010; Volume 160, pp. 63-68.

18. Okamoto, H.; Oka, M.; Tamura, I. Couplings of Thin-plate Martensites in an Fe-Ni-C Alloy. Trans. Jpn. Inst. Met. 1978, 19, 674-684. [CrossRef]

19. Stormvinter, A.; Miyamoto, G.; Furuhara, T.; Hedström, P.; Borgenstam, A. Effect of carbon content on variant pairing of martensite in Fe-C alloys. Acta Mater. 2012, 60, 7265-7274. [CrossRef]

20. Cayron, C. EBSD imaging of orientation relationships and variant groupings in different martensitic alloys and Widmanstätten iron meteorites. Mater. Charact. 2014, 94, 93-110. [CrossRef]

21. Bokros, J.; Parker, E. The mechanism of the martensite burst transformation in FeNi single crystals. Acta Metall. 1963, 11, 1291-1301. [CrossRef]

22. Thomas, G. Electron microscopy investigations of ferrous martensites. Metall. Trans. 1971, 2, 2373. [CrossRef]

(C) 2018 by the authors. Licensee MDPI, Basel, Switzerland. This article is an open access article distributed under the terms and conditions of the Creative Commons Attribution (CC BY) license (http://creativecommons.org/licenses/by/4.0/). 